\title{
Studies on positive conveying in helically channeled single screw extruders
}

\author{
L. Pan, M. Y. Jia, P. Xue*, K. J. Wang, Z. M. Jin \\ Institute of Plastic Machinery and Engineering, Beijing University of Chemical Technology, 100029 Beijing, China
}

Received 5 November 2011; accepted in revised form 20 January 2012

\begin{abstract}
A solids conveying theory called double-flight driving theory was proposed for helically channeled single screw extruders. In the extruder, screw channel rotates against static barrel channel, which behaves as cooperative embedded twin-screws for the positive conveying. They turn as two parallel arc plates, between which an arc-plate solid-plug was assumed. By analyzing the forces on the solid-plug in the barrel channel and screw channel, the boundary conditions when the solid-plug is waived of being cut off on barrel wall, were found to have the capacity of the positive conveying. Experimental data were obtained using a specially designed extruder with a helically channeled barrel in the feeding zone and a pressure-adjustable die. The effects of the barrel channel geometry and friction coefficients on the conveying mechanism were presented and compared with the experimental results. The simulations showed that the positive conveying could be achieved after optimizing extruder designs. Compared with the traditional design with the friction-drag conveying, the throughput is higher while screw torque and energy consumption are decreased. Besides, the design criteria of the barrel channel were also discussed.
\end{abstract}

Keywords: processing technologies, double-flight driving theory, positive conveying, helical barrel channels, solids conveying

\section{Introduction}

Single screw extruders were widely used as one of the basic and convenient elements to melt and convey polymer materials. It was usual to consider single-screw extruders being composed of solids conveying zone, melting zone, and melt conveying zone. Attention was here paid to the solids conveying zone closely related to the performance of the extruders.

The classical solids conveying theory, called Darnell-Mol theory, demonstrated that the friction force on the barrel internal surface must be larger than that on the screw surface for effective solids conveying and steady extrusion. An effective solution to improve feeding efficiency is to groove barrel surface along the axial direction as introduced by Menges at the Institute of Plastics Processing
(IKV) in Aachen in 1960's because the grooves greatly increases the apparent friction coefficient at the barrel-solid plug interface. Later, the effects of structural parameters on the apparent friction coefficient of the barrel were studied by Rautenbach [1], Grünschloß [2] and Potente [3]. Due to the improved barrel friction coefficient, higher pressure results in higher throughput with better stability than conventional systems, which facilitates processing high molecular weight polymer and highly filled materials [4-6]. However, such screw consumes more energy as well as greater wearing than common screws [5, 7-9].

In recent years, some advances were made on the designs of the groove structural parameters to improve the performance of the solids conveying zone. Helical grooves were first invented in the

\footnotetext{
${ }^{*}$ Corresponding author, e-mail: buct_nme@sohu.com

(c) BME-PT
} 
1970's for decreasing the deflection of the groove flow in the peripheral direction. Later, the superiority of helical grooves was testified by Kramer' experiment [10] and analyzed mathematically by Grünschloß $[11,12]$ and Miethlinger [13]. Their researches indicated that helical grooves improved the throughput much higher than axial grooves. Later, the groove adjustable continuously in geometry was used in extruders [14] and showed great advantages in controlling the solids conveying efficiency and in widening the range of materials, which was confirmed by Rauwendaal and Sikora [14], Kowalska [15] and Sikora [16]. However, the adjustable extruder has not been widely applied due to its complexity and high cost of the adjustable mechanism. The theory on solids conveying of grooved barrel extruders were also studied and modified. Rautenbach and Peiffer [17] proposed an in-depth model to determine the conveying performance of the grooved section in single-screw extruders in 1982. Potente [18] established a new throughput model in which the solids were divided into two parts, including pellets flow in the screw channel and those flow in the barrel grooves. The effects of the pressure gradient and particle size on the solids conveying angle were also analyzed by Potente [19]. Besides, optimal design of the groove helical angle was conducted by considering the effects of the pressure gradient and friction coefficient on the conveying efficiency by Rauwendaal [20]. In 2001, Potente and Phol [21] attempted to model the two-dimensional approach flow of pellets using the discrete element method. Moysey and coworkers [22-24] utilized the discrete element method to further analyze the flow characteristics of pellets with a threedimensional model. Michelangelli et al. [25] extended Potente and Monysey' efforts and investigated the effect of the average pellet size on the dynamics of the granular flow.

However, in the previous models based on the friction-drag conveying mechanism, the effects of both axial and helical grooves were only assumed to increase the mean friction coefficient of the barrel on solid pellets, by which the friction force between the barrel and the solids is the active force and the friction force between the screw and the solids is the resistant force. Shear fracture occurs on the interface between the groove and screw channel in above friction-drag conveying mechanism, so there is no positive conveying in single screw extruders. Thus, these designs based on the friction-drag conveying models have disadvantages such as larger motor load, energy consumption and rapid wear.

For solving these questions, large barrel channels, not grooves, were developed in this study to achieve the positive conveying in the solids conveying zone based on a new solids conveying theory called double-flight driving theory. In the theory, static helical barrel channels and rotating screw were regarded as a cooperative system similar to the counter-rotating twin-screws. The solids in the barrel channel and screw channel form one arc-plate solid-plug model, which is different from the traditional parallel-plate model as shown in Figure 1. From the mathematical model, the boundary condition equations for the positive conveying in single-screw extruders and the pressure equation in the solids conveying zone were established. Based on these, the design criteria for the helically channeled single screw extruders with the positive conveying were discussed, simulations were conducted, and experimental studies were carried out to verify this model.

\section{Physical model \\ 2.1. Arc-plate model}

The physical model called arc-plate model in the double-flight driving theory is shown in Figure 1. The feeding barrel sleeve is helically channeled such that pellets flow into the screw channel and fill in the barrel channels. In the modeling, one solidplug element is assumed to be composed of the solids filled in the screw channel and the connected ones limited in one helical barrel channel as Figure 1 shows. The element volume is formed by surrounding the plug element forward side and backward side along the helical screw channel, active flight

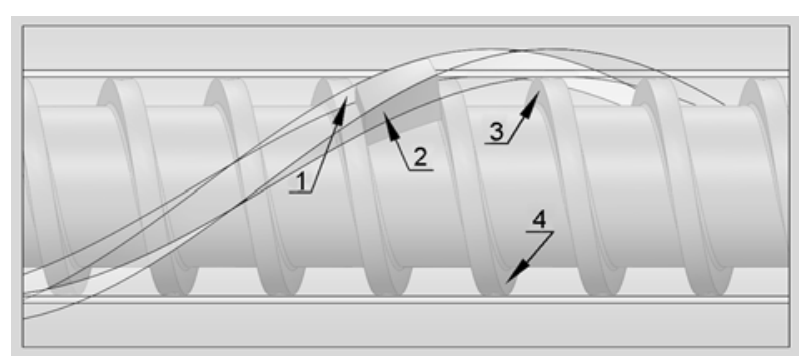

Figure 1. The arc-plate model for the screw extruder with the helically channeld barrel. 1. the active flight of the barrel channel, 2. the trailing flight of the barrel channel, 3. the trailing flight of the screw, 4. the active flight of the screw. 
and trailing flight of the screw, and active flight and trailing flight of the barrel channel. The bottom surface and the top surface of the solid-plug element are in arcs parallel to the barrel channel bottom. The other four sides of the element are in planes. Thus, the element is called one arc-plate in modeling.

If no shear fracture occurs inside the arc-plate element with the screw rotating, the element will be pushed forward along the barrel channel direction by the barrel channel and screw flight. Such solids conveying process is called the positive conveying, similar to the counter-rotating twin-screw extruder. In the positive conveying, active forces from the active flights of the barrel channel and screw channel are driving forces, and friction forces from the barrel and screw surface are resistant forces. Thus, the barrel and screw can be manufactured smoothly to have low friction heat. More importantly, the material with low friction coefficient can be steadily conveyed along the barrel channel direction by the active forces. In order to achieve the positive conveying, we carried out the theoretical analysis in Section 3 and experimental study in Sections 4 and 5.

\subsection{Basic assumptions}

(1) The granules or powder added in the hopper is compressed to form the solid-plug without internal slip in the solids conveying zone.

(2) The solid-plug closely contacts with the six surfaces: the screw channel bottom, the active and trailing flight of the screw, the barrel channel bottom, and the active and trailing flight of the barrel channel.

(3) The solid-plug is compressible and the bulk density is the function of the pressure.

(4) The pressure of the solid-plug is the function of the distance along the screw channel direction.

(5) In the barrel channel located in each screw pitch distance, the pressure gradient is ignored.

(6) The friction coefficient, in terms of the Coulomb's Law, is a constant.

(7) The barrel channels are approximated to be rectangular.

(8) The clearance between the barrel and the screw is negligible.

(9) The effects of the material gravity and the variation of the internal temperature inside the solid-plug are negligible.

\section{Mathematical model}

\subsection{Velocities and accelerations of the solid-plug}

In modeling, the barrel is assumed as the moving reference system relative to the static screw. The velocity of the solid-plug element is decomposed as shown in Figure 2. Axis $Z$ is axial direction. $\varphi$ is the screw channel helical angle at barrel wall, $\varphi_{\mathrm{N}}$ is the barrel channel helical angle in the barrel. $V_{\mathrm{b}}$ is the circumferential velocity of the barrel. The solids conveying mechanism is either the positive conveying or the friction-drag conveying. In the positive conveying, the solids move at a speed of $V_{\mathrm{S}}$ along the barrel channel direction while moving at $V_{\mathrm{r}}$ in the screw channel. In the friction-drag conveying, the solids are forwarded at $V_{\mathrm{Sf}}$ composited with $V_{\mathrm{rf}}$. It can be seen that the conveying angle of the solidplug in the positive conveying is much greater than that in the friction-drag conveying.

The inertia forces are not negligible in the solids moving, which make the solid pellets or powder into the barrel channels and further compact them. Figure 3 presents the acceleration decompositions of the solid-plug embedded in the barrel channel and screw channel.

Axis $X$ is radial direction and axis $Y$ is tangential direction. Due to the position variation of the mov-

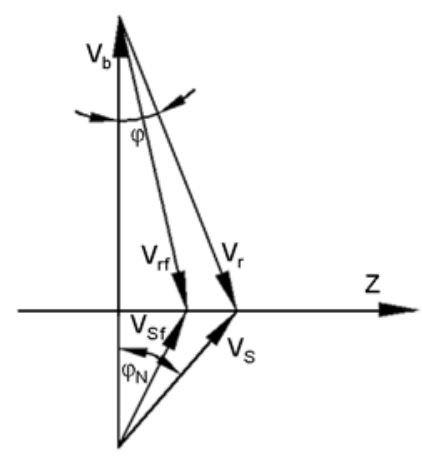

Figure 2. Velocity decompositions of the solid-plug in the positive conveying and the friction-drag conveying

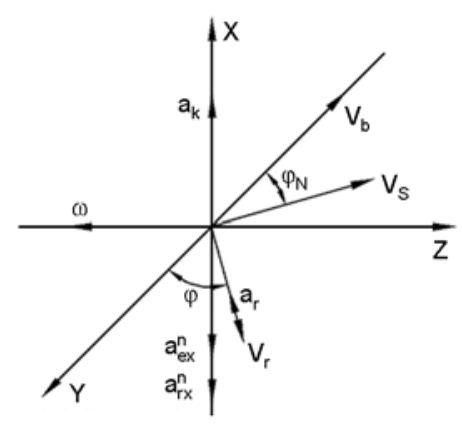

Figure 3. Acceleration decompositions of the solid-plug embedded in the barrel channel and screw channel 
ing solid-plug against rotating screw, Coriolis acceleration is generated when the solid-plug moves at $V_{\mathrm{r}}$ along the screw channel direction as shown by Equation (1):

$$
a_{\mathrm{k}}=\frac{4 V_{\mathrm{b}}^{2} \cos \varphi}{D \sin \left(\varphi_{\mathrm{N}}+\varphi\right)}
$$

The relative acceleration $a_{\mathrm{r}}$ resulting from the variation in $V_{\mathrm{r}}$ is given by Equation (2):

$a_{\mathrm{r}}=\frac{\mathrm{d} V_{\mathrm{r}}}{\mathrm{d} t}$

The velocities $V_{\mathrm{b}}$ and $V_{\mathrm{r}}$ can be decomposed into the tangential direction (Axis $Y$ ). That is, normal acceleration $a_{\mathrm{n}}$ in the radial direction (Axis $X$ ) includes $a_{\mathrm{ex}}^{\mathrm{n}}$ and $a_{\mathrm{rx}}^{\mathrm{n}}$ which can be calculated by Equation (3):

$a_{\mathrm{n}}=\frac{-2 V_{\mathrm{b}}^{2} \sin ^{2} \varphi \cos ^{2} \varphi_{\mathrm{N}}}{D \sin ^{2}\left(\varphi_{\mathrm{N}}+\varphi\right)}$

where $D$ is the outer diameter of the screw and $t$ the time.

\subsection{Force analysis}

When pushing the solid-plug forward, the two active flights can be viewed as scissors resulting in a shear stress on the interface between the part of the solidplug in the barrel channel and the rest in the screw channel. In order to determine the shear stress, the force analysis on the arc-plate model must be carried out. Figure 4 shows the forces on the solidplug. Figure 5 displays the forces on the part embedded in the barrel channel. All of the forces are included in the following:

(1) Main Inertia forces include $I_{\mathrm{n}}, I_{\mathrm{k}}$ and $I_{\mathrm{r}}$ caused by the centripetal acceleration, Coriolis acceleration and the relative acceleration, respectively.

(2) Normal forces, some of them resulting from pressure $P, P_{1}, P_{2}, P_{3}, P_{4}, P_{51}, P_{52}, P_{61}$ and $P_{62}$ per unit area on the surfaces of the solid-plug, others from friction $F_{\mathrm{FP}_{1}}, F_{\mathrm{FP}_{2}}, F_{\mathrm{FP}_{3}}, F_{\mathrm{FP}_{4}}, F_{\mathrm{FP}_{51}}$ and $F_{\mathrm{FP}_{61}}$.

$$
\begin{aligned}
& -F_{\mathrm{FP}_{1}} \cos \varphi_{\mathrm{N}} \frac{D_{\mathrm{NS}}}{2}+F_{\mathrm{FP}_{2}} \cos \varphi_{\mathrm{S}} \frac{D_{\mathrm{s}}}{2}+F_{3} \sin \varphi_{\mathrm{m}} \frac{D_{\mathrm{m}}}{2}+F_{\mathrm{P}_{3}} \sin \varphi_{\mathrm{m}} \frac{D_{\mathrm{m}}}{2}+F_{\mathrm{FP}_{3}} \cos \varphi_{\mathrm{m}} \frac{D_{\mathrm{m}}}{2}-F_{\mathrm{P}_{4}} \sin \varphi_{\mathrm{m}} \frac{D_{\mathrm{m}}}{2} \\
& +F_{\mathrm{FP}_{4}} \cos \varphi_{\mathrm{m}} \frac{D_{\mathrm{m}}}{2}-F_{5} \sin \varphi_{\mathrm{N}} \frac{D_{\mathrm{Nm}}}{2}-F_{\mathrm{P}_{51}} \sin \varphi_{\mathrm{N}} \frac{D_{\mathrm{Nm}}}{2}-F_{\mathrm{FP}_{51}} \cos \varphi_{\mathrm{N}} \frac{D_{\mathrm{Nm}}}{2}-I_{\mathrm{r}} \cos \varphi_{\mathrm{m}} \frac{D_{\mathrm{m}}}{2}-F_{\mathrm{P}_{52}} \sin \varphi_{\mathrm{N}} \frac{D_{\mathrm{m}}}{2} \\
& +F_{\mathrm{P}_{61}} \sin \varphi_{\mathrm{N}} \frac{D_{\mathrm{Nm}}}{2}-F_{\mathrm{FP}_{61}} \cos \varphi_{\mathrm{N}} \frac{D_{\mathrm{Nm}}}{2}+F_{\mathrm{P}_{62}} \sin \varphi_{\mathrm{N}} \frac{D_{\mathrm{m}}}{2}=0
\end{aligned}
$$


The force balance along the screw axis (Axis $Z$ ) gives Equation (5):

$$
\begin{aligned}
& -F_{\mathrm{FP}_{1}} \sin \varphi_{\mathrm{N}}-F_{\mathrm{FP}_{2}} \sin \varphi_{\mathrm{S}}+F_{3} \cos \varphi_{\mathrm{m}}+F_{\mathrm{P}_{3}} \cos \varphi_{\mathrm{m}}-F_{\mathrm{FP}_{3}} \sin \varphi_{\mathrm{m}}-F_{\mathrm{P}_{4}} \cos \varphi_{\mathrm{m}}-F_{\mathrm{FP}_{4}} \sin \varphi_{\mathrm{mN}}+F_{5} \cos \varphi \\
& +F_{\mathrm{P}_{51}} \cos \varphi_{\mathrm{N}}-F_{\mathrm{FP}_{51}} \sin \varphi_{\mathrm{N}}+F_{\mathrm{P}_{52}} \cos \varphi_{\mathrm{N}}+I_{\mathrm{t}} \sin \varphi_{\mathrm{m}}-F_{\mathrm{P}_{61}} \cos \varphi_{\mathrm{N}}-F_{\mathrm{FP}_{61}} \sin \varphi_{\mathrm{N}}-F_{\mathrm{P}_{62}} \cos \varphi_{\mathrm{N}}=0
\end{aligned}
$$

Combining Equations (4) and (5), it leads to Equation (6):

$F_{5}=\left(F_{\mathrm{P}_{1}}+I_{\mathrm{n}}\right) \frac{A_{2}}{A_{1}}+\left(F_{\mathrm{P}_{2}}+I_{\mathrm{k}}\right) \frac{A_{3}}{A_{1}}+F_{\mathrm{P}_{4}} \frac{A_{4}}{A_{1}}-F_{\mathrm{P}_{51}}+\left(F_{\mathrm{P}_{62}}-F_{\mathrm{P}_{52}}\right) \frac{A_{5}}{A_{1}}+F_{\mathrm{P}_{61}} \frac{A_{6}}{A_{1}}-I_{\mathrm{r}} \frac{A_{7}}{A_{1}}$

where

$$
\begin{aligned}
& A_{1}=D_{\mathrm{Nm}}\left(\cos \varphi_{\mathrm{m}}-f_{\mathrm{L}} \sin \varphi_{\mathrm{m}}\right)\left(\sin \varphi_{\mathrm{N}}+f_{\mathrm{T}} \cos \varphi_{\mathrm{N}}\right)+D_{\mathrm{m}}\left(\sin \varphi_{\mathrm{m}}+f_{\mathrm{L}} \cos \varphi_{\mathrm{m}}\right)\left(\cos \varphi_{\mathrm{N}}-f_{\mathrm{T}} \sin \varphi_{\mathrm{N}}\right) \\
& A_{2}=f_{\mathrm{T}}\left[\cos \varphi_{\mathrm{N}} D_{\mathrm{NS}}\left(f_{\mathrm{L}} \sin \varphi_{\mathrm{m}}-\cos \varphi_{\mathrm{m}}\right)+\sin \varphi_{\mathrm{N}} D_{\mathrm{m}}\left(\sin \varphi_{\mathrm{m}}+f_{\mathrm{L}} \cos \varphi_{\mathrm{m}}\right)\right] \\
& A_{3}=f_{\mathrm{L}}\left[\cos \varphi_{\mathrm{S}} D_{\mathrm{S}}\left(\cos \varphi_{\mathrm{m}}-f_{\mathrm{L}} \sin \varphi_{\mathrm{m}}\right)+\sin \varphi_{\mathrm{S}} D_{\mathrm{m}}\left(\sin \varphi_{\mathrm{m}}+f_{\mathrm{L}} \cos \varphi_{\mathrm{m}}\right)\right] \\
& A_{4}=2 D_{\mathrm{m}} f_{\mathrm{L}} \\
& A_{5}=D_{\mathrm{m}}\left[\sin \varphi_{\mathrm{N}}\left(\cos \varphi_{\mathrm{m}}-f_{\mathrm{L}} \sin \varphi_{\mathrm{m}}\right)+\cos \varphi_{\mathrm{N}}\left(\sin \varphi_{\mathrm{m}}+f_{\mathrm{L}} \cos \varphi_{\mathrm{m}}\right)\right] \\
& A_{6}=D_{\mathrm{Nm}}\left(\cos \varphi_{\mathrm{m}}-f_{\mathrm{L}} \sin \varphi_{\mathrm{m}}\right)\left(\sin \varphi_{\mathrm{N}}-f_{\mathrm{T}} \cos \varphi_{\mathrm{N}}\right)+D_{\mathrm{m}}\left(\sin \varphi_{\mathrm{m}}+f_{\mathrm{L}} \cos \varphi_{\mathrm{m}}\right)\left(\cos \varphi_{\mathrm{N}}+f_{\mathrm{T}} \sin \varphi_{\mathrm{N}}\right) \\
& A_{7}=D_{\mathrm{m}} \\
& F_{\mathrm{P}_{1}}=P_{1} b_{\mathrm{N}} b \\
& F_{\mathrm{P}_{2}}=P_{2} b_{\mathrm{N}} b_{\mathrm{S}} \\
& F_{\mathrm{P}_{4}}=P_{4} b_{\mathrm{N}} h \\
& F_{\mathrm{P}_{51}}=P_{51} b h_{\mathrm{N}} \\
& F_{\mathrm{P}_{52}}=P_{52} b_{\mathrm{m}} h \\
& F_{\mathrm{P}_{61}}=P_{61} b h_{\mathrm{N}} \\
& F_{\mathrm{P}_{62}}=P_{62} b_{\mathrm{m}} h
\end{aligned}
$$

where $A_{\mathrm{i}}(i=1,2,3,4,5,6,7)$ are the parameters that are constant for a given barrel and screw as well as material, $f_{\mathrm{T}}$ the friction coefficient of the solids on the barrel, $f_{\mathrm{L}}$ the friction coefficient of the solids on the screw, $D_{\mathrm{NS}}$ the barrel channel root diameter, $D_{\mathrm{Nm}}$ the mean barrel channel diameter, $D_{\mathrm{S}}$ the screw root diameter, $D_{\mathrm{m}}$ the mean screw diameter, $b_{\mathrm{N}}$ the barrel channel width, $b$ the screw channel width at barrel wall, $b_{\mathrm{S}}$ the screw channel width at screw root, $b_{\mathrm{m}}$ the mean screw channel width, $h_{\mathrm{N}}$ the barrel channel depth, $h$ the screw channel depth, $\varphi_{\mathrm{S}}$ the

$$
\begin{aligned}
& -F_{\mathrm{FP}_{1}} \cos \varphi_{\mathrm{N}} \frac{D_{\mathrm{NS}}}{2}+\hat{\mathrm{o}} b_{\mathrm{N}} b \sin \alpha \frac{D_{\mathrm{N}}}{2}-F_{5} \sin \varphi_{\mathrm{N}} \frac{D_{\mathrm{Nm}}}{2}-F_{\mathrm{P}_{51}} \sin \varphi_{\mathrm{N}} \frac{D_{\mathrm{Nm}}}{2}-F_{\mathrm{FP}_{51}} \cos \varphi_{\mathrm{N}} \frac{D_{\mathrm{Nm}}}{2}+F_{\mathrm{P}_{61}} \sin \varphi_{\mathrm{N}} \frac{D_{\mathrm{Nm}}}{2} \\
& -F_{\mathrm{FP}_{61}} \cos \varphi_{\mathrm{N}} \frac{D_{\mathrm{Nm}}}{2}=0
\end{aligned}
$$


where $\alpha$ is the angle between the direction of the interface shear stress ô and the axial direction as shown in Figure 6 and the barrel channel diameter at barrel wall.

The equilibrium of forces on the solid-plug part embedded in the barrel channel along the screw axis (Axis $Z$ ) is:

$\begin{aligned}- & F_{\mathrm{FP}_{1}} \sin \varphi_{\mathrm{N}}+\hat{\mathrm{o}} b_{\mathrm{N}} b \cos \alpha+F_{5} \cos \varphi_{\mathrm{N}}-F_{\mathrm{P}_{51}} \cos \varphi_{\mathrm{N}}- \\ & F_{\mathrm{FP}_{51}} \sin \varphi_{\mathrm{N}}-F_{\mathrm{P}_{61}} \cos \varphi_{\mathrm{N}}-F_{\mathrm{FP}_{61}} \sin \varphi_{\mathrm{N}}=0\end{aligned}$

By substituting $F_{5}$ in Equation (8) into Equation (7), the interface shear stress ô amounts to:

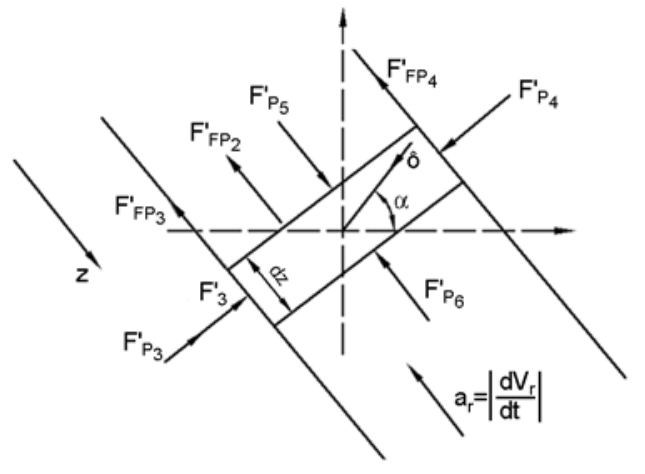

Figure 6. Motion and forces on the differential element in the screw channel

$$
\hat{\mathrm{o}}=\frac{\sqrt{F_{\mathrm{P}}^{2} f_{\mathrm{T}}^{2}+F_{5}^{2}\left(1+f_{\mathrm{T}}^{2}\right)+4 F_{\mathrm{P}_{5}}^{2} f_{\mathrm{T}}^{2}+2 F_{\mathrm{P}_{1}} F_{5} f_{\mathrm{T}}^{2}+4 F_{\mathrm{P}_{51}} F_{5} f_{\mathrm{T}}^{2}+4 F_{\mathrm{P}_{1}} F_{\mathrm{P}_{51}} f_{\mathrm{T}}^{2}}}{b_{\mathrm{N}} b}
$$

\subsection{Boundary conditions for positive conveying}

In order to have the capacity of the positive conveying, the shear stress ô must be less than the internal friction force per unit area at the interface between the part of the solid-plug embedded in the barrel channel and the rest in the screw channel so that the plug could move as a whole. Therefore, the first boundary condition equation is:

$\hat{\mathrm{o}} \leq P f_{\mathrm{i}}$

Substituting Equation (9) into Equation (10), it becomes Equation (11):

$$
\frac{\sqrt{F_{\mathrm{P}_{1}}^{2} f_{\mathrm{T}}^{2}+F_{5}^{2}\left(1+f_{\mathrm{T}}^{2}\right)+4 F_{\mathrm{P}_{51}}^{2} f_{\mathrm{T}}^{2}+2 F_{\mathrm{P}_{1}} F_{5} f_{\mathrm{T}}^{2}+4 F_{\mathrm{P}_{51}} F_{5} f_{\mathrm{T}}^{2}+4 F_{\mathrm{P}_{1}} F_{\mathrm{P}_{5}} f_{\mathrm{T}}^{2}}}{b_{\mathrm{N}} b} \leq P f_{\mathrm{i}}
$$

or

$$
F_{\mathrm{P}_{1}}^{2} f_{\mathrm{T}}^{2}+F_{5}^{2}\left(1+f_{\mathrm{T}}^{2}\right)+4 F_{\mathrm{P}_{51}}^{2} f_{\mathrm{T}}^{2}+2 F_{\mathrm{P}_{1}} F_{5} f_{\mathrm{T}}^{2}+4 F_{\mathrm{P}_{51}} F_{5} f_{\mathrm{T}}^{2}+4 F_{\mathrm{P}_{1}} F_{\mathrm{P}_{51}} f_{\mathrm{T}}^{2} \leq\left(P b_{\mathrm{N}} b f_{\mathrm{i}}\right)^{2}
$$

When Equation (12) is divided by $F_{\mathrm{P}}^{2} f_{\mathrm{T}}^{2}$, the first boundary condition equation can approximately be simplified as follows:

$$
\left(\frac{F_{5}}{F_{\mathrm{P}_{1}}}\right)^{2}\left[1+\frac{1}{f_{\mathrm{T}}^{2}}\right]+\frac{F_{5}}{F_{\mathrm{P}_{1}}}\left[2+4 \frac{F_{\mathrm{P}_{51}}}{F_{\mathrm{P}_{1}}}\right]+\left[2 \frac{F_{\mathrm{P}_{51}}}{F_{\mathrm{P}_{1}}}+1\right]^{2} \leq\left(\frac{f_{\mathrm{i}}}{f_{\mathrm{T}}}\right)^{2}
$$

where $f_{\mathrm{i}}$ is internal friction coefficient in solids, and $\frac{F_{5}}{F_{\mathrm{P}_{1}}}=\frac{A_{2}}{A_{1}}+\frac{A_{3}}{A_{1}}+\frac{h}{b} \frac{A_{4}}{A_{1}}-\frac{h_{\mathrm{N}}}{b_{\mathrm{N}}}+\frac{h_{\mathrm{N}}}{b_{\mathrm{N}}} \frac{A_{6}}{A_{1}}$ $\frac{F_{\mathrm{P}_{51}}}{F_{\mathrm{P}_{1}}}=\frac{h_{\mathrm{N}}}{b_{\mathrm{N}}}$

From Equation (13), it can be seen that the boundary condition is the function of the friction coefficients and structural parameters. $h_{\mathrm{N}} / b_{\mathrm{N}}$ is defined as the barrel channel aspect ratio. It is known from
Equation (13) that the lower the friction coefficient of the barrel surface is, the easier the positive conveying can be achieved. More importantly, when the solid-plug is positively conveyed by the two active forces of the barrel and screw channel flights, the extruder has an operating mode where the solids conveying is independent of the friction coefficient on the barrel surface. Therefore, different friction coefficients on the barrel surface only induce different energy consumption and pressure peak at the end of the solids conveying zone and don't affect 
the solids conveying process. If the friction coefficient on the barrel surface becomes nil, the solidplug is also conveyed along the barrel channel helical angle. However, in the case of the friction-drag conveying, an effective solids conveying is dependent on a sufficient high friction coefficient on the barrel surface. The greater the friction coefficient of the barrel surface is, the steadier the friction-drag conveying is. As a result, higher energy consumption is required for the friction-drag conveying, compared to that in terms of the positive conveying.

\subsection{Pressure equation}

Continuity equation and kinematic equation are used to analyze the motion and forces of the differential element in the screw channel. The pressure equation in the solids conveying zone can be obtained after resolving the two equations.

A down-channel differential element is displayed in Figure 6, where $z$ is the distance along the screw channel direction. The continuity equation is given by Equation (14):

$$
\frac{\partial \rho}{\partial t}+V_{\mathrm{r}} \frac{\partial \rho}{\partial z}+\rho \frac{\partial V_{\mathrm{r}}}{\partial z}=0
$$

Where the material density is varied along the screw channel, given by Equation (15) [26]:

$\rho=\rho_{\mathrm{m}}-\left(\rho_{\mathrm{m}}-\rho_{0}\right) \mathrm{e}^{-\mathrm{C}_{0} \mathrm{P}}$

Inserting Equation (15) into Equation (14), the continuity equation becomes Equation (16):

$$
\frac{\partial P}{\partial t}+V_{\mathrm{r}} \frac{\partial P}{\partial z}+\frac{1}{C_{0}}\left[\frac{\rho_{\mathrm{m}} \mathrm{e}^{\mathrm{C}_{0} \mathrm{P}}}{\rho_{\mathrm{m}}-\rho_{0}}-1\right] \frac{\partial V_{\mathrm{r}}}{\partial z}=0
$$

where $\rho$ is the density under pressure $P$ at time $t, \rho_{\mathrm{m}}$ the density under utmost pressure, $\rho_{0}$ the bulk density under the atmospheric pressure and $C_{0}$ a coefficient.

The kinematics equation can be determined by the application of the force balance to the differential element in Figure 6 in both the down-channel direction and the direction perpendicular to the screw channel.
The equilibrium of forces in the direction perpendicular to the screw channel is given by Equation (17):

$F^{\prime}{ }_{3}=\hat{o} \cos (\alpha-\varphi) b d z_{\mathrm{b}}$

The equilibrium of forces in the down-channel direction gives Equation (18):

$F^{\prime}{ }_{\mathrm{PP}_{2}}+F^{\prime}{ }_{\mathrm{PP}_{3}}+F^{\prime}{ }_{\mathrm{FP}_{4}}+\left(F_{\mathrm{P}_{6}}^{\prime}-F_{\mathrm{P}_{5}}^{\prime}\right)$

$-\hat{\mathrm{o}} \sin (\alpha-\varphi) b d z_{\mathrm{b}}=m^{\prime}\left|a_{\mathrm{r}}\right|$

where $m^{\prime}$ is the mass of the differential element in the screw channel, $F^{\prime}{ }_{\mathrm{P}_{3}}, F^{\prime}{ }_{\mathrm{P}_{4}}, F_{\mathrm{P}_{5}}^{\prime}, F_{\mathrm{P}_{6}}^{\prime}, F^{\prime}{ }_{\mathrm{FP}_{2}}, F^{\prime}{ }_{\mathrm{FP}_{3}}$, $F^{\prime}{ }_{\mathrm{FP}_{4}}$ the normal forces on the differential element in the screw channel and $F^{\prime}{ }_{3}$ the active force on the differential element from the active flight of the screw.

Substituting Equation (17) into Equation (18) leads the kinematic equation to be expressed as shown by Equation (19):

$$
\frac{\partial P}{\partial z}+P D_{1}+\rho\left[V_{\mathrm{r}}^{2} D_{2}+D_{3}\left(\frac{\mathrm{d} V_{\mathrm{r}}}{\mathrm{d} t}\right)\right]=0
$$

where $D_{\mathrm{i}}(i=1,2,3)$ are the parameters that are constant for a given barrel and screw as well as material.

The boundary conditions for the movement of solids are:

$$
\begin{array}{ll}
\left.V_{\mathrm{r}}(z, t)\right|_{\mathrm{z}=0}=V_{0}=V_{\mathrm{b}} \cos \varphi, & \left.V_{\mathrm{r}}(z, t)\right|_{\mathrm{t}=0}=V_{0} \\
\left.P(z, t)\right|_{\mathrm{z}=0}=P_{0}, & \left.P(z, t)\right|_{\mathrm{t}=0}=\bar{P}
\end{array}
$$

where $V_{0}$ is the inlet velocity of solids along the screw channel direction, $P_{0}$ the inlet pressure and $\bar{P}$ the average pressure.

Equations (16) and (19) are resolved using Laplace transformation, Laplace ultimate theory and dimensional transformation [27]. The pressure on the solid-plug along the screw channel direction can be written as shown by Equation (20):

$$
P=P_{0} \cdot \mathrm{e}^{\frac{-\beta \mathrm{z}}{\mathrm{L}}}+\frac{\gamma^{2} \rho_{\mathrm{m}} V_{0}^{2} D_{2}}{D_{1}} \cdot\left(\mathrm{e}^{\frac{-\beta \mathrm{z}}{\mathrm{L}}}-1\right)
$$

where $\gamma$ is the modification factor, $L$ the length of the barrel channel along the axial direction (Axis $Z$ ) and

$$
\beta=\frac{\rho_{0} L D_{1}}{\rho_{0}-\gamma^{2} C_{0} \rho_{\mathrm{m}} V_{0}^{2} D_{3}\left(\rho_{\mathrm{m}}-\rho_{0}\right)} D_{1}=\frac{\sin \varphi\left(b_{\mathrm{S}} f_{\mathrm{L}} \frac{\sin \varphi_{\mathrm{m}}}{\sin \varphi_{\mathrm{S}}}+2 f_{\mathrm{L}} h\right)+\sin \varphi_{\mathrm{m}}\left(b C_{1}+b_{\mathrm{S}} C_{2}+h C_{3}+\frac{b h_{\mathrm{N}} C_{5}}{b_{\mathrm{N}}}\right)}{b_{\mathrm{m}} h\left[\sin \varphi+\sin \varphi_{\mathrm{m}} C_{4}\right]}
$$




$$
\begin{aligned}
& D_{2}=\frac{\sin \varphi}{\sin \varphi+\sin \varphi_{\mathrm{m}} C_{4}}\left[\frac{2 \sin \varphi_{\mathrm{m}}\left(1+\frac{b h_{\mathrm{N}}}{b_{\mathrm{m}} h} C_{\mathrm{n}}\left(\sin \varphi \cot \varphi_{\mathrm{N}}\right)^{2}\right.}{D \sin \varphi}+\left(\frac{\sin \varphi_{\mathrm{m}}\left(1+\frac{b h_{\mathrm{N}}}{b_{\mathrm{m}} h}\right) C_{\mathrm{k}}}{\sin \varphi}+f_{\mathrm{L}}\right) \frac{4 \cos \varphi \sin \left(\varphi_{\mathrm{N}}+\varphi\right)}{D \sin \varphi_{\mathrm{N}}}\right] \\
& D_{3}=\frac{\sin \varphi}{\sin \varphi+\sin \varphi_{\mathrm{m}} C_{4}}\left[1-\frac{\sin \varphi_{\mathrm{m}}\left(1+\frac{b h_{\mathrm{N}}}{b_{\mathrm{m}} h}\right) C_{\mathrm{t}}}{\sin \varphi}\right] \\
& C_{1}=C_{\mathrm{n}}=\left(f_{\mathrm{L}} \cos \varphi+\sin \varphi\right)\left[f_{\mathrm{T}} \sin \varphi_{\mathrm{N}}+\frac{A_{2}}{A_{1}}\left(f_{\mathrm{T}} \sin \varphi_{\mathrm{N}}-\cos \varphi_{\mathrm{N}}\right)\right] \\
& +\left[f_{\mathrm{T}} \cos \varphi_{\mathrm{N}} D_{\mathrm{NS}}+\frac{A_{2}}{A_{1}} D_{\mathrm{Nm}}\left(\sin \varphi_{\mathrm{N}}+f_{\mathrm{T}} \cos \varphi_{\mathrm{N}}\right)\right] \frac{f_{\mathrm{L}} \sin \varphi-\cos \varphi}{D_{\mathrm{N}}} \\
& C_{2}=C_{\mathrm{k}}=\left(f_{\mathrm{L}} \cos \varphi+\sin \varphi\right) \frac{A_{3}}{A_{1}}\left(f_{\mathrm{T}} \sin \varphi_{\mathrm{N}}-\cos \varphi_{\mathrm{N}}\right)+\frac{A_{3}}{A_{1}} D_{\mathrm{Nm}}\left(\sin \varphi_{\mathrm{N}}+f_{\mathrm{T}} \cos \varphi_{\mathrm{N}}\right) \frac{f_{\mathrm{L}} \sin \varphi-\cos \varphi}{D_{\mathrm{N}}} \\
& C_{3}=\left(f_{\mathrm{L}} \cos \varphi+\sin \varphi\right) \frac{A_{4}}{A_{1}}\left(f_{\mathrm{T}} \sin \varphi_{\mathrm{N}}-\cos \varphi_{\mathrm{N}}\right)+\frac{A_{4}}{A_{1}} D_{\mathrm{Nm}}\left(\sin \varphi_{\mathrm{N}}+f_{\mathrm{T}} \cos \varphi_{\mathrm{N}}\right) \frac{f_{\mathrm{L}} \sin \varphi-\cos \varphi}{D_{\mathrm{N}}} \\
& C_{\mathrm{t}}=\left(f_{\mathrm{L}} \cos \varphi+\sin \varphi\right) \frac{A_{7}}{A_{1}}\left(\cos \varphi_{\mathrm{N}}-f_{\mathrm{T}} \sin \varphi_{\mathrm{N}}\right)+\frac{A_{7}}{A_{1}} D_{\mathrm{Nm}}\left(\sin \varphi_{\mathrm{N}}+f_{\mathrm{T}} \cos \varphi_{\mathrm{N}}\right) \frac{f_{\mathrm{L}} \sin \varphi-\cos \varphi}{D_{\mathrm{N}}} \\
& C_{5}=\left(f_{\mathrm{L}} \cos \varphi+\sin \varphi\right)\left[\left(\cos \varphi_{\mathrm{N}}+f_{\mathrm{T}} \sin \varphi_{\mathrm{N}}\right)+\frac{A_{6}}{A_{1}}\left(f_{\mathrm{T}} \sin \varphi_{\mathrm{N}}-\cos \varphi_{\mathrm{N}}\right)\right] \\
& \left.\left.C_{\mathrm{N}}-\sin \varphi_{\mathrm{N}}\right)+\frac{A_{6}}{A_{1}}\left(\sin \varphi_{\mathrm{N}}+f_{\mathrm{T}} \cos \varphi_{\mathrm{N}}\right)\right] \frac{f_{\mathrm{L}} \sin \varphi-\cos \varphi}{\left.D_{\mathrm{N}} \cos \varphi+\sin \varphi\right) \frac{A_{5}}{A_{1}}\left(f_{\mathrm{T}} \sin \varphi_{\mathrm{N}}-\cos \varphi_{\mathrm{N}}\right)+\frac{A_{5}}{A_{1}} D_{\mathrm{Nm}}\left(\sin \varphi_{\mathrm{N}}+f_{\mathrm{T}} \cos \varphi_{\mathrm{N}}\right) \frac{f_{\mathrm{L}} \sin \varphi-\cos \varphi}{D_{\mathrm{N}}}} \\
&
\end{aligned}
$$

Equation (20) indicates that the pressure on the solids conveying zone is not only the function of the friction coefficients, material density and structural parameters, but also the function of the circumferential velocity. More importantly, it also discloses that the pressure can be well established along the screw channel direction when the inlet pressure is equal to zero, which can not be effectively explained by the Darnell-Mol theory.

In Equation (20), when the parameter $\beta$ is larger than zero, the pressure is minus along the screw channel direction, which is no practical significance. If the parameter $\beta$ is negative, the pressure is positive and can be built along the screw channel direction. Therefore, the second boundary equation can be obtained and showed as Equation (21):

$$
\beta=\frac{\rho_{0} L D_{1}}{\rho_{0}-\gamma^{2} C_{0} \rho_{\mathrm{m}} V_{0}^{2} D_{3}\left(\rho_{\mathrm{m}}-\rho_{0}\right)}<0
$$

Equations (13) and (21) are used to determine whether the given parameters of the barrel channel and screw are good or not so that the optimal barrel channel and screw channel can be designed for larger positive conveying.

To summarize, the important components of the double-flight driving theory were established: two positive conveying boundary equations and the pressure equation in the solids conveying zone. 
Sections 4 and 5 will present the experiments used to verify the double-flight driving theory with our extruder.

\section{Experimental \\ 4.1. Apparatus}

One helically channeled extruder was specially designed and manufactured to study the positive conveying mechanism as shown in Figure 7. The extruder can be used to measure the pressure value on-line at the end of the solids conveying zone and the throughput that was only composed of the solids conveying zone and equipped with one detachable feeding barrel sleeve, one screw and one pressureadjustable die. One taper barrel sleeve with two rows of small circular holes shown in Figure 8 is installed between the screw and diversion cone. Two rows of circular holes with their axis leaning to screw axis are evenly and alternately arranged along the circumferential direction. Their gross area is equal to the total area of the barrel channels and screw channel. While the extruder working, small circular holes are used as the outlets of the die for extruding solids so that solids pellets are extruded evenly in the peripheral direction. Therefore, the motion trace of the solids is approaching in the real case. The outlet area of the die is constant to make sure the steady extrusion.

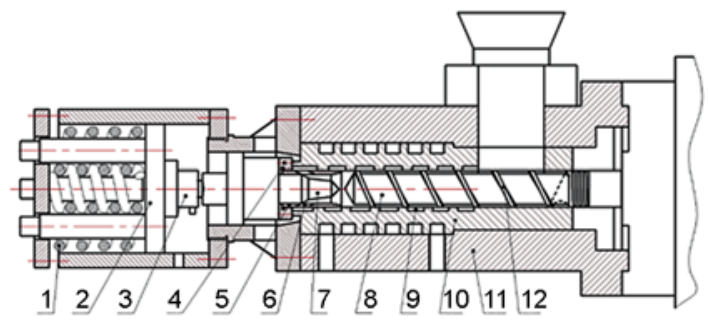

Figure 7. A schematic diagram of the extruder specially designed for the experiments. 1. spring, 2. spring bumper, 3. weighing sensor, 4. flange, 5. taper sleeve, 6. outlets, 7. diversion cone, 8. screw channel, 9. barrel channel, 10. feeding sleeve, 11. barrel, 12. screw.
Besides, in order to discuss the effects of the geometrical parameters on the positive conveying mechanism and the performance of the solids conveying zone, two helically channeled sleeves with different barrel channel widths and two screws with different pitches were made, as shown in Figure 9. The mean barrel channel helical angles are both $50^{\circ}$ with eight barrel channels in the two helically channeled sleeves. The screws are of diameter $45 \mathrm{~mm}$ and

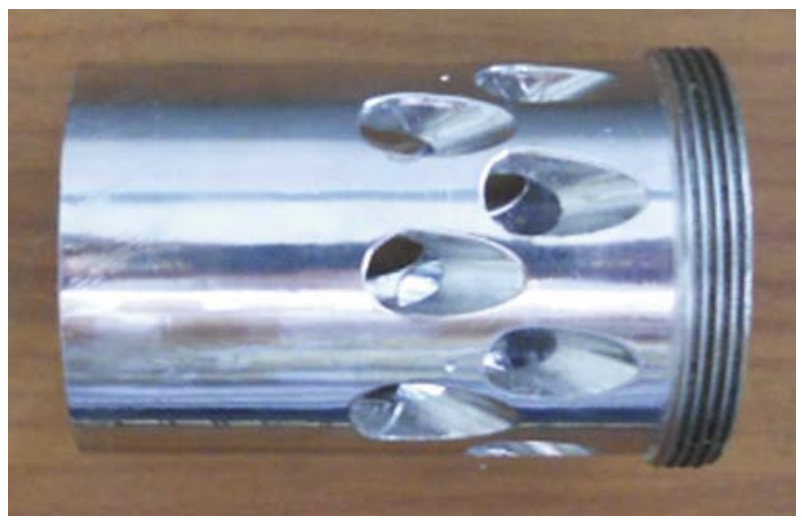

Figure 8. A taper sleeve with two rows of small circular holes

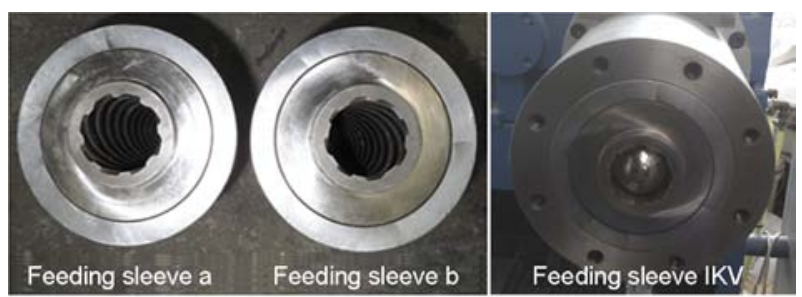

a)

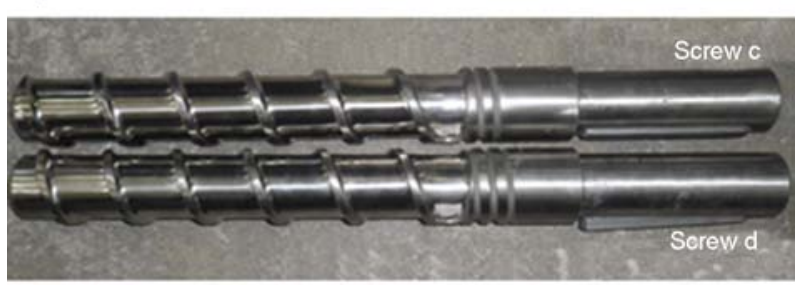

b)

Figure 9. A schematic diagram of the feeding sleeves and screws. (a) two helically channeled sleeves with different barrel channel widths and one axially grooved sleeve, (b) two screws with different pitches.

Table 1. Geometrical parameters of the solids conveying zone of the experimental extruder

\begin{tabular}{|l|c|c|c|c|c|c|}
\hline $\begin{array}{c}\text { Experimental } \\
\text { components }\end{array}$ & Thread number & Lead & Length & $\begin{array}{c}\text { Barrel channel } \\
\text { depth } \\
{[\mathbf{m m}]}\end{array}$ & $\begin{array}{c}\text { Barrel channel } \\
\text { width } \\
{[\mathbf{m m}]}\end{array}$ & $\begin{array}{c}\text { Flight width } \\
\text { [mm] }\end{array}$ \\
\hline Sleeve $\boldsymbol{a}$ & 8 & From 180 to 156 & 261 & 2.0 & From 8.5 to 7.7 & 5.5 \\
\hline Sleeve $\boldsymbol{b}$ & 8 & From 180 to 156 & 261 & 2.0 & From 7.0 to 6.2 & 7.0 \\
\hline Sleeve $\boldsymbol{I} \boldsymbol{K} \boldsymbol{V}$ & 8 & - & 261 & 1.5 & 8 & 9.6 \\
\hline Screw $\boldsymbol{c}$ & 1 & 45 & 261 & 3.2 & - & 4.5 \\
\hline Screw $\boldsymbol{d}$ & 1 & From 45 to 39 & 261 & 3.2 & - & 4.5 \\
\hline
\end{tabular}


Table 2. Physical properties of LDPE used in the experiments

\begin{tabular}{|c|c|c|c|c|c|c|}
\hline Material & $\begin{array}{c}\text { Melt flow index } \\
{[\mathbf{g} / \mathbf{1 0} \mathbf{~ m i n}]}\end{array}$ & $\begin{array}{c}\text { Bulk density } \\
{\left[\mathbf{k g} / \mathbf{m}^{3}\right]}\end{array}$ & $\begin{array}{c}\text { Density under } \\
\text { utmost pressure } \\
{\left[\mathbf{k g} / \mathbf{m}^{3}\right]}\end{array}$ & $\begin{array}{c}\text { External friction } \\
\text { coefficient } \\
\left(\boldsymbol{f}_{\mathrm{L}}=\boldsymbol{f}_{\mathrm{T}}\right)\end{array}$ & $\begin{array}{c}\text { Internal friction } \\
\text { coefficient } \\
\left(\boldsymbol{f}_{\mathrm{i}}\right)\end{array}$ & $\begin{array}{c}\text { Mean diameter } \\
\text { of particles } \\
{[\mathbf{m m}]}\end{array}$ \\
\hline LDPE & 7.5 & 485 & 920 & 0.13 & 0.45 & $1.6-2.0$ \\
\hline
\end{tabular}

have a length to diameter ratio of 5.8. In addition, the feeding sleeve $\boldsymbol{I} \boldsymbol{K} \boldsymbol{V}$ with axial grooves was also designed to compare the effects of the geometrical parameters on the solids conveying mechanism. The basic geometrical parameters of the experimental extruder are given in Table 1.

\subsection{Materials}

Low-density Polyethylene (LDPE, LD607 type) from Beijing Yanshan Plant was used with the physical properties listed in Table 2. In the experiments, the friction coefficients were assumed to be constant.

\section{Results and discussions}

\subsection{Boundary conditions for positive conveying}

Two critical condition equations (Equation (22) and (23)) for the positive conveying can be obtained from the two boundary condition Equations (13) and (21).

When $\hat{\mathrm{o}}=P f_{\mathrm{i}}$, the first critical condition Equation can be determined as follows:

$X_{1}=\left(\frac{F_{5}}{F_{\mathrm{P}_{1}}}\right)^{2}\left(1+\frac{1}{f_{\mathrm{T}}^{2}}\right)+\frac{F_{5}}{F_{\mathrm{P}_{1}}}\left(2+4 \frac{F_{\mathrm{P}_{51}}}{F_{\mathrm{P}_{1}}}\right)+\left(2 \frac{F_{\mathrm{P}_{51}}}{F_{\mathrm{P}_{1}}}+1\right)^{2}-\left(\frac{f_{\mathrm{i}}}{f_{\mathrm{T}}}\right)^{2}=0$

If $\beta=0$, the second critical condition equation can be written as Equation (23):

$X_{2}=\frac{\rho_{0} L D_{1}}{\rho_{0}-\gamma^{2} C_{0} \rho_{\mathrm{m}} V_{0}^{2} D_{3}\left(\rho_{\mathrm{m}}-\rho_{0}\right)}=0$

When the geometrical parameters of the screw, the physical parameters of material and the screw rotation are known, the unknown barrel channel aspect ratio $h_{\mathrm{N}} / b_{\mathrm{N}}$ is resolved from Equations (22) and (23) as a function of the barrel channel helical angle. Thus, two critical curves can be drawn.

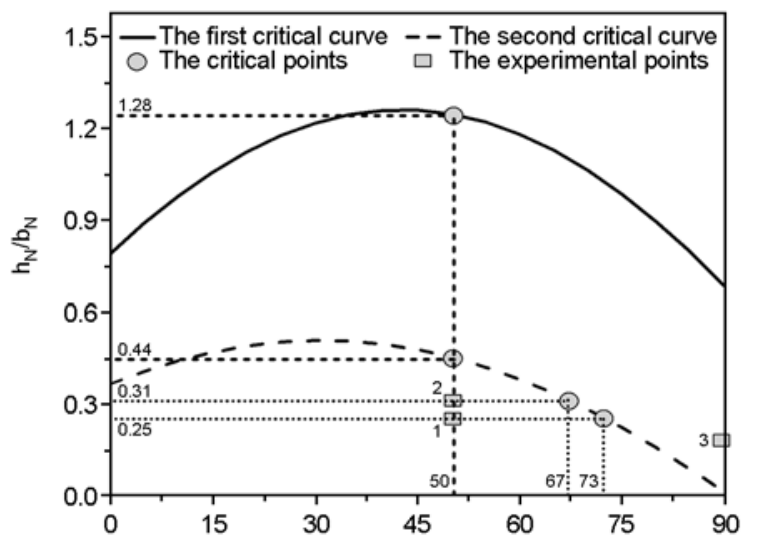

a)

$\varphi_{\mathrm{N}}\left[^{\circ}\right]$
In order to compare with the experimental data directly, theoretical simulations are carried out using the same parameters in Table 1 . The two critical curves for the given screw $\boldsymbol{c}$ and $\boldsymbol{d}$ and material can be drawn at the screw rotation speed of $40 \mathrm{rpm}$, shown in Figure 10. It can be seen that only when the values of the barrel channel aspect ratio and barrel channel helical angle are both simultaneously less than their corresponding critical points in the critical curves, the boundary condition Equations (13) and (21) can be both maintained. There-

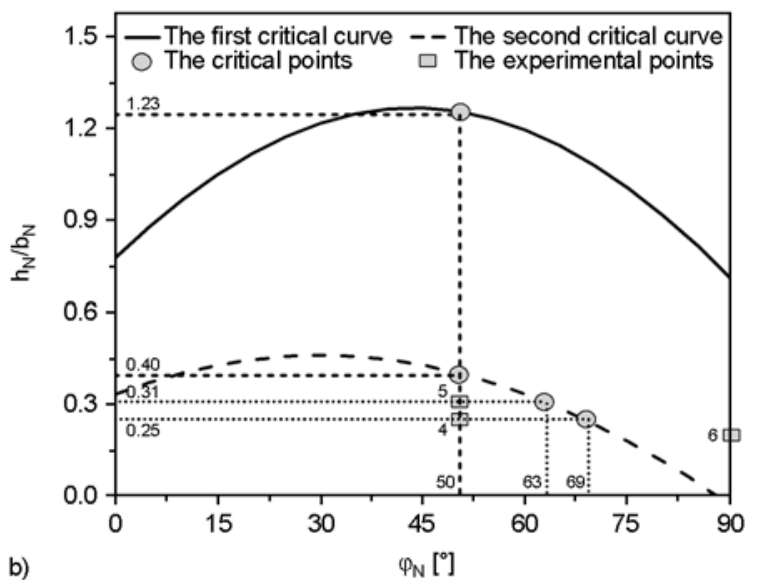

Figure 10. Predicted critical curves for positive conveying and experimental data. (a) the predicted critical curves and experimental values 1,2 and 3 for the case with screw $c$, (b) the predicted critical curves and experimental values 4, 5 and 6 for the case with screw $\boldsymbol{d} .1,2,3,4,5$ and 6 are the experimental values for the combination $\boldsymbol{c}$ - $\boldsymbol{a}$ of the screw $\boldsymbol{c}$ and the feeding sleeve $\boldsymbol{a}, \boldsymbol{c}-\boldsymbol{b}, \boldsymbol{c}-\boldsymbol{I K} \boldsymbol{V}, \boldsymbol{d}-\boldsymbol{a}, \boldsymbol{d}-\boldsymbol{b}$ and $\boldsymbol{d}-\boldsymbol{I} \boldsymbol{K} \boldsymbol{V}$. 
fore, it can be concluded that, to have the capacity of the positive conveying, both the barrel channel aspect ratio and barrel channel helical angle must be beneath the two critical curves so that they are simultaneously less than their corresponding critical points. Otherwise, it is the friction-drag conveying. On the one hand, it can be seen from Figure 10a and $10 \mathrm{~b}$ that the four experimental points 1 with the combination $\boldsymbol{c}-\boldsymbol{a}$ of the screw $\boldsymbol{c}$ and the feeding sleeve $\boldsymbol{a}, 2$ with $\boldsymbol{c}-\boldsymbol{b}, 4$ with $\boldsymbol{d}-\boldsymbol{a}$ and 5 with $\boldsymbol{d}-\boldsymbol{b}$ are all in the area beneath the two critical curves, which means that the positive conveying works in the four experiments. As for the experimental point 3 with the combination $\boldsymbol{c}-\boldsymbol{I} \boldsymbol{K} \boldsymbol{V}$ of the screw $\boldsymbol{c}$ and the feeding sleeve $\boldsymbol{I K} \boldsymbol{V}$, and point 6 with $\boldsymbol{d}-\boldsymbol{I} \boldsymbol{K} \boldsymbol{V}$, they both fall out of the area beneath the two critical curves, so it is the friction-drag conveying in the two experiments. Positive conveying can be achieved in axial grooves only in the case of the minimum aspect ratio (zero), which has no practical significance. Thus, the feeding sleeve $\boldsymbol{I} \boldsymbol{K} \boldsymbol{V}$ with axial grooves can not achieve positive conveying in practice.

On the other hand, it can be calculated by Equations (22) and (23) that the critical values of the barrel channel aspect ratio for the two experimental points 1 and 2 are 1.27 and 0.44 corresponding to the first and second critical curves respectively in Figure 10a while those for the two experimental points 4 and 5 are 1.23 and 0.4 respectively in Figure $10 \mathrm{~b}$ with the given barrel channel helical angle of $50^{\circ}$ in feeding sleeve $\boldsymbol{a}$ and $\boldsymbol{b}$. Based on the theoretical analysis of two critical curves, the positive conveying can be achieved if the maximum values of the barrel channel aspect ratio are less than 0.44 in Figure 10a and 0.4 in Figure 10b with the given barrel channel helical angle of $50^{\circ}$. Otherwise, the frictiondrag conveying works. Similarly, The maximum barrel channel helical angle in Figure 10a must be less than $73^{\circ}$ for the point 1 to get positive conveying with the given value of the barrel channel aspect ratio of 0.25 in the feeding sleeve a and that is $67^{\circ}$ for the point 2 with the given value of the barrel channel aspect ratio of 0.31 in the feeding sleeve $\boldsymbol{b}$. Besides, the maximum barrel channel helical angle in Figure 10b must be less than $69^{\circ}$ for the point 4 with the given value of the barrel channel aspect ratio of 0.25 in the feeding sleeve $\boldsymbol{a}$ and that is $63^{\circ}$ for the point 5 with the given value of the barrel channel aspect ratio of 0.31 in the feeding sleeve $\boldsymbol{b}$.

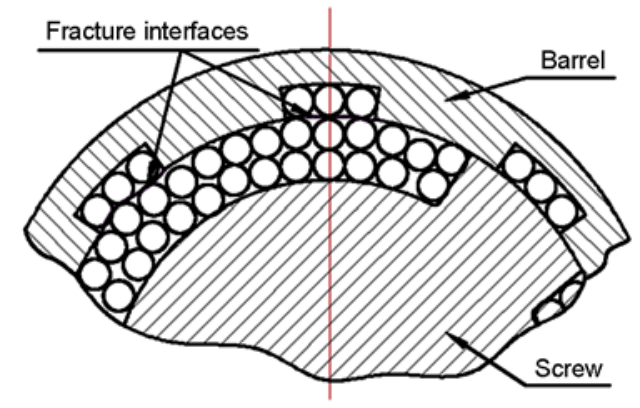

Figure 11. A schematic diagram of fracture interfaces inside the solid-plug

Therefore, the critical curves define such two characteristics in the positive conveying mechanism.

One characteristic is that the maximum barrel channel helical angle can be determined when the barrel channel aspect ratio is known. It can be explained by the fact that the interface shear stress is raised remarkably with the increasing barrel channel helical angle. When the interface shear stress is bigger than the internal friction force, the solid-plug can be cut off at the interface as shown in Figure 11, and then the conveying is not the positive conveying. The other characteristic is that the maximum barrel channel aspect ratio also can be obtained when the barrel channel helical angle is given. It can be known from above theoretical studies that the friction forces from the barrel channel are the resistant forces and the active forces of the screw and barrel channel flight are the driving forces when the solidplug in the barrel channel and screw channel is positively conveyed. However, it is worthwhile to mention that the active forces of the screw and barrel channel flight are both derived from the interface shear stress. Therefore, the deeper the barrel channel depth is, the greater the resistant force is. The wider the barrel channel width is, the greater the active force is. If the maximum barrel channel aspect ratio is exceeded, the active forces will not be enough so that the solid-plug will be cut off at the interface and there is no barrel channel conveyance. This is also the reason why the frictiondrag conveying prevails in the experimental points 3 and 6 . Thus, single screw extruders with the positive conveying can be designed by the two critical curves.

Figure 12 shows the theoretical simulations about the effects of different external friction coefficients on the critical curves using the same parameters as 


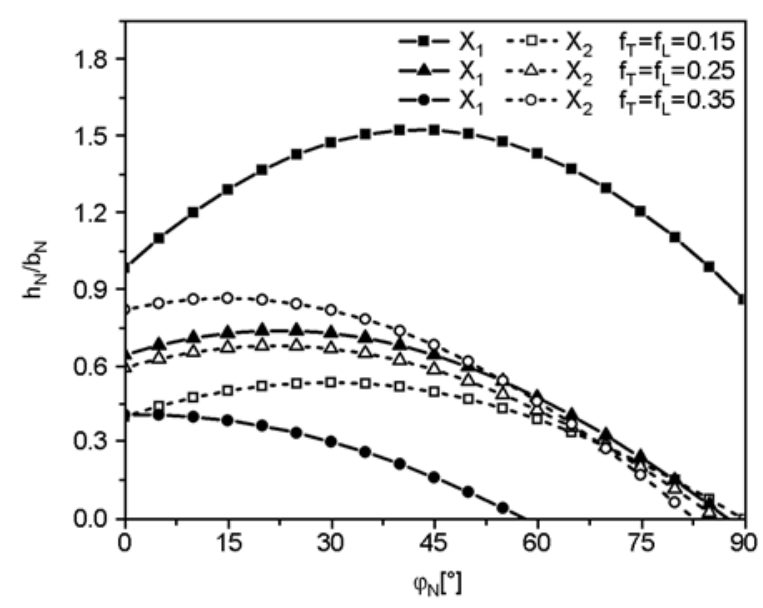

Figure 12. Effect of different external friction coefficients on the critical curves

screw $\mathrm{c}$ at the screw rotation speed of $40 \mathrm{rpm}$ at a constant internal friction coefficient of 0.6.

It can be found from Figure 12 that different external friction coefficients lead to the variation of the two critical curves corresponding to different critical barrel channel aspect ratios and barrel channel helical angles. When the external friction coefficient increases, the location of the first critical curve descends while the location of the second critical curve ascends. Besides, it can also be seen that the greater the external friction coefficient is, the smaller the available area for positive conveying beneath the two critical curves is. It can be concluded that big external friction coefficients can result in the transformation from the positive con-

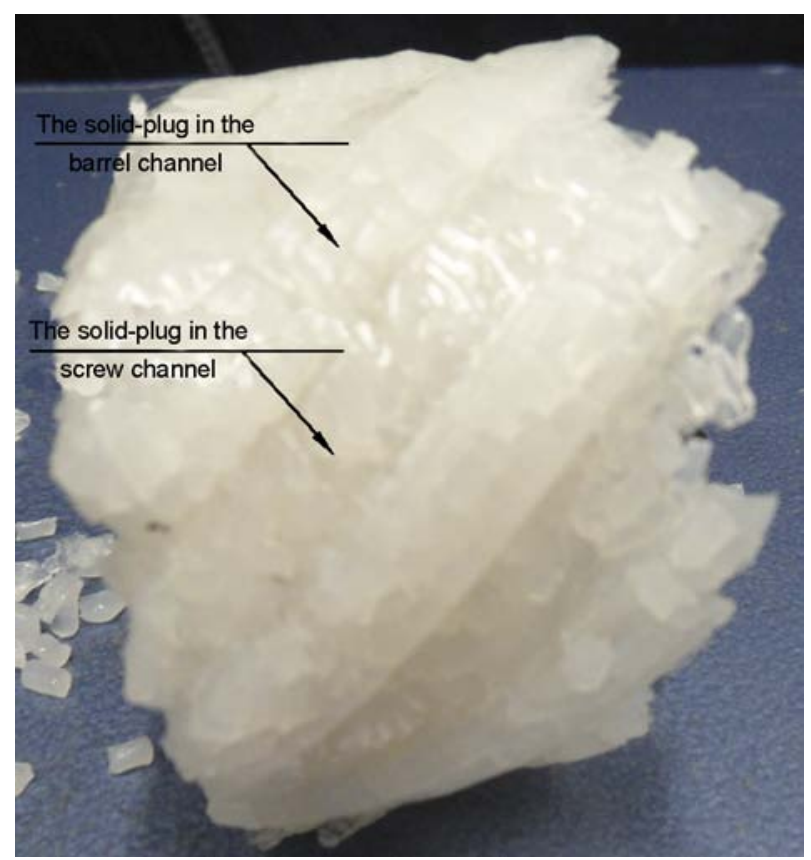

Figure 13. A whole solid-plug sample in a steady solids conveying process in the experimental extruder veying to the friction-drag conveying, which is due to the fact that great friction coefficient of the barrel surface induces high resistance force for barrel channel conveyance in the positive conveying so that the solid-plug is cut off at barrel wall.

Figure 13 shows a whole solid-plug sample in a steady solids conveying process using the experimental extruder with the combination $\boldsymbol{c}-\boldsymbol{a}$ at a screw rotation speed of $40 \mathrm{rpm}$. When the extruder runs steadily, we stop the screw suddenly to disassemble the pressure-adjustable die and to subsequently peel off the polymer sample located in the screw head. It can be seen from the sample in Figure 13 that the solids in the barrel channel and screw channel were extruded as a whole solid-plug indicating no internal circumferential shear fracture at the shear interface and the positive conveying prevailing. This experiment showed the typical positive conveying using the combination $\boldsymbol{c}-\boldsymbol{a}$, which is close to the predicted results from the point 1 in Figure 10a.

\subsection{Pressure distribution}

In order to verify the positive conveying mechanism further, some theoretical simulations about the effects of the geometrical parameters on the pressure distribution in the solids conveying zone were carried out at a screw rotation speed of $40 \mathrm{rpm}$. Moreover, the on-line measured results of experimental pressure were compared with theoretical analysis.

The pressure distribution curves in the solids conveying zone with different solids conveying mechanisms and external friction coefficients are presented in Figure 14. The first experiment was made

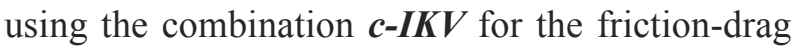
conveying. The second experiment was carried out using the combination $\boldsymbol{c}$ - $\boldsymbol{a}$ for the positive conveying. It can be found from Figure 14 that the simulated pressure distribution in the friction-drag conveying by the Darnell-Mol theory increases sharply and is seriously deflected from the experimental data. The reason may be that the density of the solid-plug is assumed to be invariable and the acceleration of the solid-plug is not considered. In contrast, the simulated pressure distribution in the positive conveying by the present model increases linearly and is well consistent with the experimental data at the end of the solids conveying zone. This confirms that the double-flight driving theory can 


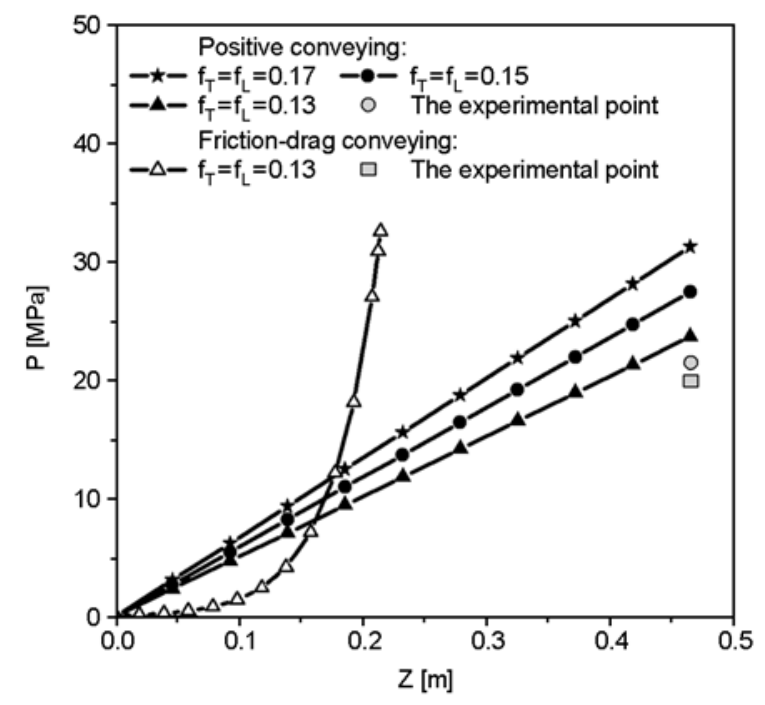

Figure 14. Pressure distribution in the solids conveying zone with different mechanisms and different friction coefficients

better describe the pressure distribution in the solids conveying zone than the Darnell-Mol theory. Besides, it is worthwhile to notice that the two experimental values in different solids conveying mechanisms are not obviously different indicating that both positive and friction-drag conveying mechanisms also have the good building pressure capability. In addition, it also can be seen that bigger external friction coefficients induce higher pressure increase in the solids conveying zone, which is consistent with researches of Fang et al. [28].

Effect of the barrel channel helical angle on the pressure value at the end of the solids conveying zone is shown in Figure 15. The experiment was performed with the combination $\boldsymbol{c}$ - $\boldsymbol{a}$. The pressure value at the end of the solids conveying zone was

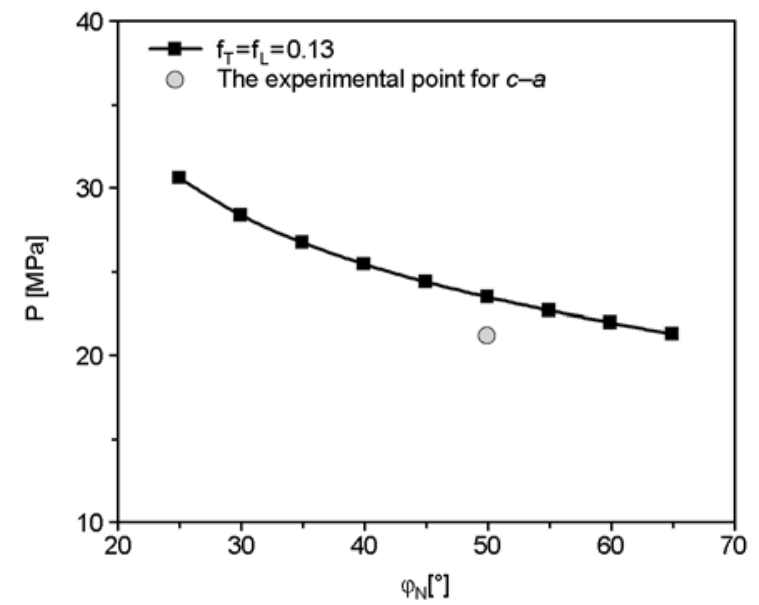

Figure 15. Effect of the barrel channel helical angle on the pressure value at the end of the solids conveying zone found to decrease with the increasing barrel channel helical angle. In the case of the positive conveying, the conveying angle of the solid-plug in the screw channel is equal to the barrel channel helical angle. Thus, high barrel channel helical angle induces big conveying angle of the solid-plug in the screw channel, which improves the conveying rate of the solids in the screw channel resulting in low pressure value. In addition, it also can be known from the figure that the experimental value approaches to the theoretical data with the given external friction coefficient of 0.13 , which confirms the accuracy of the theory.

Figure 16 displays the effect of the screw pitch on the pressure value at the end of the solids conveying zone, including the experimental data for the combinations $\boldsymbol{c}-\boldsymbol{a}$ and $\boldsymbol{d}-\boldsymbol{a}$, respectively. It can be found that the simulated pressure value at the end of the solids conveying zone is raised with the increasing screw pitch size because the throughput in the screw channel is higher at a constant barrel channel conveying angle, which is helpful to compact solidplug. The predicted values are consistent with the experimental data. However, such tendency is not that obvious at high barrel channel helical angle, which can be due to the fact that increasing barrel channel helical angle results in the decrease of the pressure value.

Figure 17 presents the predicted pressure value and experimental data at the end of the solids conveying zone for the combinations $\boldsymbol{c}-\boldsymbol{a}$ and $\boldsymbol{c}-\boldsymbol{b}$. The simulated pressure values at the end of the solids conveying zone vary insignificantly with different barrel channel widths, as is consistent with the experimen-

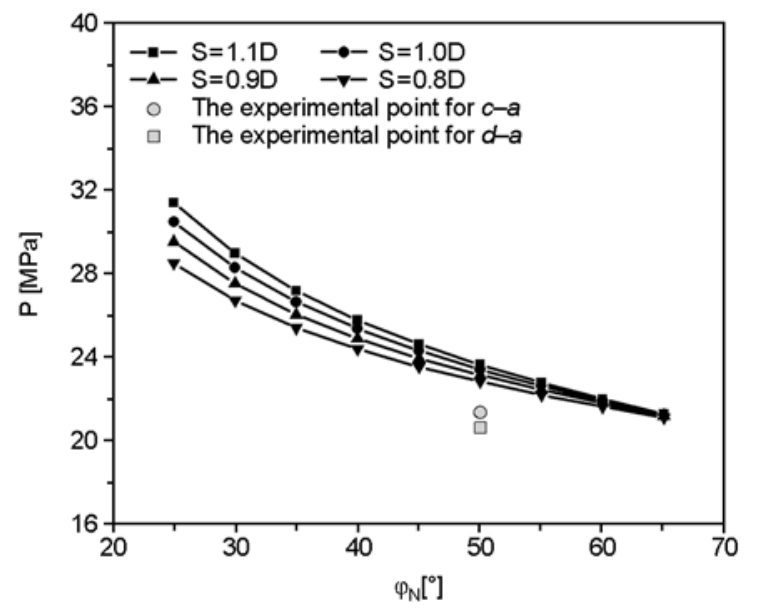

Figure 16. Effect of the screw pitch size on the pressure value at the end of the solids conveying zone 


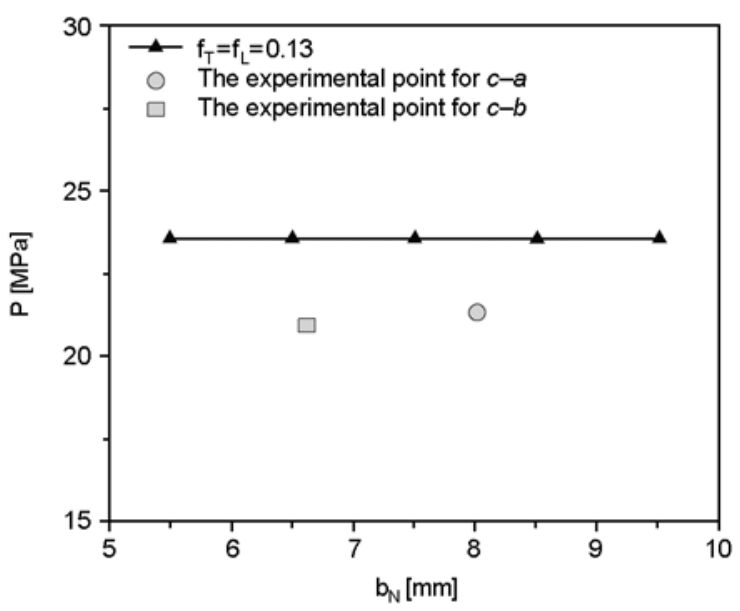

Figure 17. Effect of the barrel channel width on the pressure value at the end of the solids conveying zone

tal data. This can well be understood by comparing the difference between the double-flight driving theory and the Darnell-Mol theory. In the case of the friction-drag conveying, the barrel friction force is the active force and the mean friction coefficient of the barrel is raised greatly with the increasing barrel channel width by some previous reports [1$3,13]$, which results in high pressure value at the end of the solids conveying zone. In contrast, all the friction forces from the barrel and screw surface are resistance forces in the double-flight driving theory for the positive conveying and the solid-plug is only pushed forward along the barrel channel helical angle by the two active flights of the barrel channel and screw. Therefore, the solids conveyance in the screw channel in the case of the positive conveying is hardly affected by the variation of the barrel channel width with a steady pressure value at the end of the solids conveying zone.

\subsection{Throughput and energy consumption}

According to above theoretical analysis on the positive conveying mechanism, the advantages of high throughput and low energy consumption can be revealed when one extruder works with the positive conveying. Experimental verifications for the positive conveying mechanism were also implemented from the view of throughput and energy consumption.

In the case of the positive conveying, the solids embedded in the barrel channel and screw channel as a whole plug are conveyed. Thus, taking the material velocity along the axial direction and the barrel channel and screw channel area around the screw axis into consideration, the total throughput $m$ is given by Equation (24):

$$
m=n \pi \overline{D_{\mathrm{m}}}\left[\frac{\pi}{4}\left(D_{\mathrm{NS}}^{2}-D_{\mathrm{S}}^{2}\right)-M \frac{e_{1} h}{\sin \varphi_{\mathrm{m}}}-N \frac{e_{2} h_{\mathrm{N}}}{\sin \varphi_{\mathrm{N}}}\right] \frac{\sin \varphi \sin \varphi_{\mathrm{N}}}{\sin \left(\varphi+\varphi_{\mathrm{N}}\right)} \rho_{0} \cdot 60
$$

where $n$ is the screw rotation, $\overline{D_{\mathrm{m}}}$ the average diameter of the solid-plug, $e_{1}$ and $e_{2}$ the flight land width of the screw and barrel channel respectively, and $M$ and $N$ the thread numbers of the screw channel and barrel channel respectively.

Figure 18 describes the throughput measured and simulated for the combinations of $\boldsymbol{c}-\boldsymbol{a}, \boldsymbol{c}-\boldsymbol{b}$ and $\boldsymbol{c}-\boldsymbol{I K} \boldsymbol{V}$. It can be seen that the measured and simulated throughput data of the extrusion system are all improved greatly with the increasing screw rotation for the three combinations. The simulated throughput by the positive conveying mechanism for the combinations $\boldsymbol{c}-\boldsymbol{a}$ and $\boldsymbol{c}-\boldsymbol{b}$ are both close to the experimental values, which shows that the positive conveying mechanism is achieved during extrusion. Based on the positive conveying mechanism, greater barrel channel width induces larger barrel channel conveying square, which is consistent with the fact that the experimental throughput using the feeding sleeve $\boldsymbol{a}$ with great barrel channel width is larger than that of the feeding sleeve $\boldsymbol{b}$ with small barrel channel width. Thus, the positive conveying mechanism is confirmed by the above experiments. In addition, it also can be seen that the measured

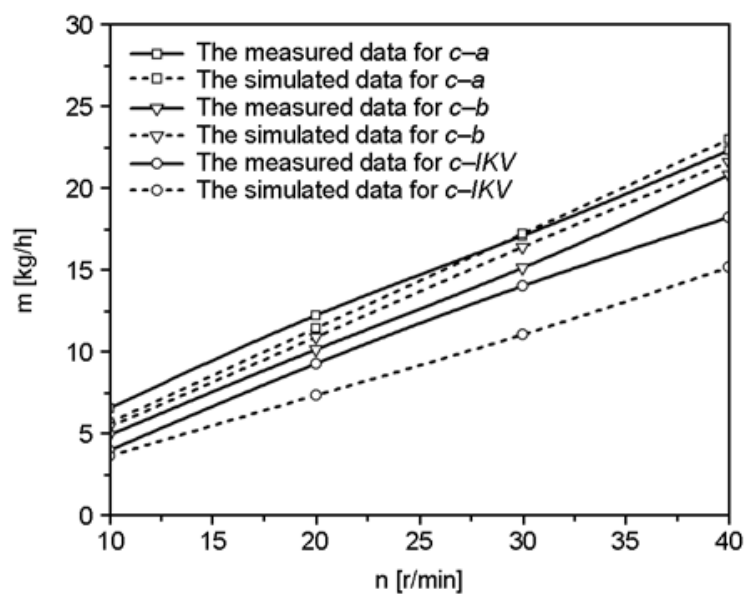

Figure 18. The measured and simulated throughput with the combinations $\boldsymbol{c}-\boldsymbol{a}, \boldsymbol{c}-\boldsymbol{b}$ and $\boldsymbol{c}-\boldsymbol{I} \boldsymbol{K} \boldsymbol{V}$ 
Table 3. Experimental electric currents, total power and theoretically useful power at different screw rotations

\begin{tabular}{|c|c|c|c|c|c|c|c|c|c|}
\hline \multirow{3}{*}{$\begin{array}{c}\mathbf{n} \\
{[\mathbf{r} / \mathbf{m i n}]}\end{array}$} & \multicolumn{3}{|c|}{ Combination $c-a$} & \multicolumn{3}{|c|}{ Combination $c-b$} & \multicolumn{3}{|c|}{ Combination $c$-IKV } \\
\hline & \multicolumn{2}{|c|}{ Experimental data } & \multirow{2}{*}{ 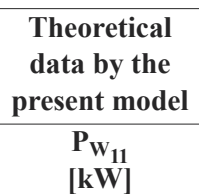 } & \multicolumn{2}{|c|}{ Experimental data } & \multirow{2}{*}{\begin{tabular}{|c|}
$\begin{array}{c}\text { Theoretical } \\
\text { data by the } \\
\text { present model }\end{array}$ \\
$\mathbf{P}_{\mathbf{W}_{22}}$ \\
{$[\mathbf{k W}]$}
\end{tabular}} & \multicolumn{2}{|c|}{ Experimental data } & \multirow{2}{*}{\begin{tabular}{|c|}
$\begin{array}{c}\text { Theoretical data } \\
\text { by Darnel-Mol } \\
\text { theory }\end{array}$ \\
$\mathbf{P}_{\mathrm{W}_{33}}$ \\
{$[\mathrm{~kW}]$}
\end{tabular}} \\
\hline & $\begin{array}{c}\mathbf{I}_{1} \\
{[\mathbf{A}]}\end{array}$ & $\begin{array}{c}\mathbf{P}_{\mathbf{W}_{1}} \\
{[\mathbf{k W}]}\end{array}$ & & $\begin{array}{c}\mathbf{I}_{2} \\
{[\mathbf{A}]}\end{array}$ & $\begin{array}{c}\mathbf{P}_{\mathbf{W}_{2}} \\
{[\mathrm{~kW}]}\end{array}$ & & $\begin{array}{c}\mathbf{I}_{3} \\
{[\mathbf{A}]}\end{array}$ & $\begin{array}{c}\mathbf{P}_{\mathbf{W}_{3}} \\
{[\mathbf{k W}]}\end{array}$ & \\
\hline 10 & 3.7 & 1.41 & 1.13 & 3.3 & 1.24 & 0.94 & 5.2 & 1.95 & 56.96 \\
\hline 20 & 6.1 & 2.31 & 1.96 & 5.2 & 1.96 & 1.68 & 8.8 & 3.35 & 113.92 \\
\hline 30 & 8.6 & 3.25 & 2.93 & 7.6 & 2.89 & 2.52 & 11.3 & 4.29 & 170.88 \\
\hline 40 & 11.6 & 4.41 & 3.80 & 10.4 & 3.95 & 3.45 & 14.5 & 5.51 & 227.82 \\
\hline
\end{tabular}

throughput with the combination $\boldsymbol{c}-\boldsymbol{I} \boldsymbol{K} \boldsymbol{V}$ is less than those of the combinations $\boldsymbol{c}-\boldsymbol{a}$ and $\boldsymbol{c}-\boldsymbol{b}$. This is because the solid-plug experiences fracture at barrel wall and the friction-drag conveying prevails during extrusion, which results in the decrease of the barrel channel conveyance. Besides, it is worth noticing that the simulated results for the combination $\boldsymbol{c - I K V}$ are seriously deflected from the measured results because only the solids in the screw channel are considered and those in the barrel channel are neglected when the friction-drag conveying prevailing in the Darnell-Mol theory.

The experimental electric currents, total power and theoretically useful power at different screw rotations for the three combinations $\boldsymbol{c}-\boldsymbol{a}, \boldsymbol{c}-\boldsymbol{b}$ and $\boldsymbol{c}-\boldsymbol{I K} \boldsymbol{V}$ are listed in Table 3.

$I_{1}, I_{2}$ and $I_{3}$ are the experimental electric currents for the three combinations $\boldsymbol{c}-\boldsymbol{a}, \boldsymbol{c}-\boldsymbol{b}$ and $\boldsymbol{c}-\boldsymbol{I} \boldsymbol{K} \boldsymbol{V}$ respectively and $P_{\mathrm{W}_{1}}, P_{\mathrm{W}_{2}}$ and $P_{\mathrm{W}_{3}}$ are the total power corresponding to $I_{1}, I_{2}$ and $I_{3} . P_{\mathrm{W}_{11}}$ and $P_{\mathrm{W}_{22}}$ are the theoretically useful power for the combinations $\boldsymbol{c}$-a and $\boldsymbol{c}-\boldsymbol{b}$ by the positive conveying theory and $P_{\mathrm{W}_{33}}$ the theoretically useful power for the combination of $\boldsymbol{c}-\mathbf{I} \boldsymbol{K} \boldsymbol{V}$ by the Darnell-Mol theory.

The variation of the electric current reflects the change of the total power in the motor because the total power of a three-phase induction motor is proportional to the electric current. In the present model for the combinations $\boldsymbol{c}-\boldsymbol{a}$ and $\boldsymbol{c}-\boldsymbol{b}$, only the useful power of $P_{\mathrm{W}_{11}}$ and $P_{\mathrm{W}_{22}}$ for solids conveyance are calculated. That results in the theoretical data less than the experimental data of $P_{\mathrm{W}_{1}}$ and $P_{\mathrm{W}_{2}}$, respectively. It can be noticed that because the deviation between the experimental and theoretical data still keeps in a small range with the increase of the screw rotation, the experimental data shows the actual energy consumption indicating that the positive conveying mechanism works during extrusion.
In addition, the comparison of $P_{\mathrm{W}_{11}}$ and $P_{\mathrm{W}_{22}}$ also shows that the simulated useful power in the positive conveying mechanism is enhanced with the increasing barrel channel width for the combinations $\boldsymbol{c}-\boldsymbol{a}$ and $\boldsymbol{c}-\boldsymbol{b}$, which can also be explained by the fact that the active forces of the barrel channel and screw flight are improved with the increase of the barrel channel width resulting in higher friction heat on the active flights of the barrel channel and screw. It also can be seen that the experimental value of the total power for the combination $\boldsymbol{c}-\mathbf{I K} \boldsymbol{V}$ with friction-drag conveying is remarkably higher than those for the combinations $\boldsymbol{c}-\boldsymbol{a}$ and $\boldsymbol{c}$ - $\boldsymbol{b}$ with positive conveying. This may be due to that the solid-plug in the barrel channel and screw channel experiences fracture at barrel wall for the combination $\boldsymbol{c}-\boldsymbol{I} \boldsymbol{K} \boldsymbol{V}$ when the friction-drag conveying mechanism prevails during extrusion resulting in high friction heat. From Table 3, it also can be found that the theoretical value of $P_{\mathrm{W}_{33}}$ for the combination $\boldsymbol{c}-\boldsymbol{I} \boldsymbol{K} \boldsymbol{V}$ is much greater than the experimental value due to the high pressure value predicted by the Darnell-Mol theory.

\section{Conclusions}

A solids conveying theory called double-flight driving theory was proposed for helically channeled single screw extruders where the solids embedded in the barrel channel and screw channel behaving as a whole solid-plug were pushed forward along the barrel channel helical angle direction by active flights of the barrel channel and screw. In order to achieve positive conveying in the double-flight driving theory, two boundary conditions for the positive conveying were determined by analyzing force equations and pressure equation. On the basis of the theoretical and experimental studies on the helically channeled feed zone, the positive convey- 
ing mechanism was confirmed by the results meas- $\quad V_{\text {rf }}$ ured and simulated by our theory.

From the boundary condition equations, the maximum barrel channel helical angle could be determined with a given barrel channel aspect ratio. Vice Versa, the maximum barrel channel aspect ratio also could be obtained with a given barrel channel helical angle for positive conveying. The external friction coefficients determine if the conveying is the positive conveying or the friction-drag conveying. More importantly, an extruder with the positive conveying can be designed from the analysis of boundary condition equations.

Compared with the sharp increase of the pressure value in the friction-drag conveying theory of the Darnel-Mol Model, the pressure distribution in the positive conveying increases linearly. In addition, the pressure value at the end of the solids conveying zone in the positive conveying is higher at lower barrel channel helical angle, higher external friction coefficients and larger screw pitch size. However, the effect of the barrel channel width is not significant. Therefore, the ability to build pressure in the solids conveying zone can be effectively controlled. The extruder designed on the positive conveying mechanism showed higher throughput and lower energy consumption than that on the friction-drag conveying mechanism. Besides, bigger barrel channel width is helpful to obtain a steady solids conveying and high throughput in the case of the positive conveying.

\section{Acknowledgements}

The authors would like to acknowledge the support of the National Natural Science Foundation of China (No. 50873014).

\section{Nomenclature}

$X \quad$ radial direction

$Y \quad$ tangential direction

$Z \quad$ axial direction

$z \quad$ distance along screw channel direction

$V_{\mathrm{b}} \quad$ circumferential velocity of barrel

$V_{\mathrm{S}} \quad$ velocity along barrel channel direction in positive conveying

$V_{\mathrm{r}} \quad$ velocity along screw channel direction in positive conveying

$V_{\text {Sf }} \quad$ velocity along barrel channel direction in friction-drag conveying
$V_{\text {rf }} \quad$ velocity along screw channel direction in friction-drag conveying

$V_{0} \quad$ inlet velocity of solids along screw channel direction

$n \quad$ screw rotation

$a_{\mathrm{n}} \quad$ normal acceleration

$a_{\mathrm{k}} \quad$ coriolis acceleration

$a_{\mathrm{r}} \quad$ relative acceleration

$I_{\mathrm{n}}, I_{\mathrm{k}}, I_{\mathrm{r}}$ inertia forces

$P, P_{1}, P_{2}, P_{3}, P_{4}, P_{51}, P_{52}, P_{61}, P_{62}$

pressure

$P_{0} \quad$ inlet pressure

$\bar{P} \quad$ average pressure

$F_{\mathrm{P}_{1}}, F_{\mathrm{P}_{2}}, F_{\mathrm{P}_{3}}, F_{\mathrm{P}_{4}}, F_{\mathrm{P}_{51}}, F_{\mathrm{P}_{52}}, F_{\mathrm{P}_{61}}, F_{\mathrm{P}_{62}}$ normal forces on the solid-plug resulting from pressure

$F_{\mathrm{FP}_{1}}, F_{\mathrm{FP}_{2}}, F_{\mathrm{FP}_{3}}, F_{\mathrm{FP}_{4}}, F_{\mathrm{FP}_{51}}, F_{\mathrm{FP}_{61}}$ normal forces on the solid-plug resulting from friction

$F_{3} \quad$ active force on solid-plug from active flight of screw

$F_{5} \quad$ active force on solid-plug from active flight of barrel channel

$F^{\prime}{ }_{\mathrm{P}_{3}}, F^{\prime}{ }_{\mathrm{P}_{4}}, F^{\prime}{ }_{\mathrm{P}_{5}}, F^{\prime} \mathrm{P}_{6}$ normal forces on differential element in screw channel resulting from pressure

$F^{\prime} \mathrm{FP}_{2}, F_{\mathrm{FP}_{3}}^{\prime}, F_{\mathrm{FP}_{4}}^{\prime}$

normal forces on differential element in screw channel resulting from friction

$F^{\prime}{ }_{3} \quad$ active force on differential element from active flight of screw

ô interface shear stress between part of solidplug in barrel channel and the rest in screw channel

$A_{\mathrm{i}}(i=1,2,3,4,5,6,7)$

parameters that are constant for given barrel and screw as well as material

$D_{\mathrm{i}}(i=1,2,3)$

parameters that are constant for given barrel and screw as well as material

$f_{\mathrm{T}} \quad$ friction coefficient of solids on barrel

$f_{\mathrm{L}} \quad$ friction coefficient of solids on screw

$f_{\mathrm{i}} \quad$ internal friction coefficient in solids

$t \quad$ time

$\rho \quad$ density at pressure $P$ at time $t$

$\rho_{\mathrm{m}} \quad$ density at utmost pressure

$\rho_{0} \quad$ bulk density at atmospheric pressure

$C_{0} \quad$ coefficient of material 
angle between direction of interface shear stress and axial direction

$\gamma$

$L \quad$ length of barrel channel along axial direction

$D_{\mathrm{N}} \quad$ barrel channel diameter at barrel wall

$D_{\mathrm{NS}} \quad$ barrel channel root diameter

$D_{\mathrm{Nm}} \quad$ mean barrel channel diameter

$D \quad$ outer diameter of screw

$D_{\mathrm{S}} \quad$ screw root diameter

$D_{\mathrm{m}} \quad$ mean screw diameter

$\overline{D_{\mathrm{m}}} \quad$ average diameter of the solid-plug

$b_{\mathrm{N}} \quad$ barrel channel width

$b \quad$ screw channel width at barrel wall

$b_{\mathrm{S}} \quad$ screw channel width at screw root

$b_{\mathrm{m}} \quad$ mean screw channel width

$h_{\mathrm{N}} \quad$ barrel channel depth

$h \quad$ screw channel depth

$\varphi_{\mathrm{N}} \quad$ barrel channel helical angle

$\varphi \quad$ screw channel helical angle at barrel wall

$\varphi_{\mathrm{S}} \quad$ screw channel helical angle at screw root

$\varphi_{\mathrm{m}} \quad$ mean screw channel helical angle

$e_{1} \quad$ flight land width of screw

$e_{2} \quad$ flight land width of barrel channel

$M \quad$ thread numbers of screw channel

$N \quad$ thread numbers of barrel channel

$m \quad$ total throughput in solids conveying zone

$m^{\prime} \quad$ mass of differential element in screw channel in Figure 6

$I_{\mathrm{i}}(i=1,2,3)$

experimental electric current

$P_{\mathrm{W}_{\mathrm{i}}}(i=1,2,3)$

total power

$P_{\mathrm{W}_{\mathrm{ii}}}(i=1,2,3)$

theoretically useful power

\section{References}

[1] Rautenbach R.: Model tests on the transport of plastics powders in the feed section of single-screw extruders. Kunststoffe-German Plastics, 69, 377-380 (1979).

[2] Grünschloß E.: Calculation of the mean coefficient of barrel friction in grooved feed sections. KunststoffeGerman Plastics, 74, 405-409 (1984).

[3] Potente H.: Methods of calculating grooved extruder feed sections. Kunststoffe-German Plastics, 75, 439441 (1985).

[4] Franzkoch B., Menges G.: Grooved forced-feeding zones can improve extruder performance. Plastics Engineering, 34, 51-54 (1978).
[5] Rautenbach R., Peiffer H.: Throughput and torque characteristics of grooved feed sections in singlescrew extruders. Kunststoffe-German Plastics, 72, 262-266 (1982).

[6] Davis B. A., Gramann P. J., Del P. Norieqa E. M., Osswald T. A.: Grooved feed single screw extruders Improving productivity and reducing viscous heating effects. Polymer Engineering and Science, 38, 11991204 (1998).

DOI: $10.1002 /$ pen.10288

[7] Menges G., Feistkorn W., Fischbach G.: Hot-operated grooved bushes increase the output and reduce the energy consumption of single screw extruders. Kunststoffe-German Plastics, 74, 695-699 (1984).

[8] Grünschloß E.: Process improvements in single-screw extruders with grooved feed sections. KunststoffeGerman Plastics, 75, 850-854 (1985).

[9] Schuele H., Fritz H. G.: Minimizing the abrasive wear in the feed zone of grooved barrel extruders. Kunststoffe-German Plastics, 77, 387-393 (1987).

[10] Kramer A.: Experience in using extruders with grooved feed zones. Kunststoffe-German Plastics, 78, 21-26 (1988)

[11] Grünschloß E.: A new style single screw extruder with improved plastification and output power. International Polymer Processing, 17, 291-300 (2002).

[12] Grünschloß E.: A powerful universal plasticating system for single-screw-extruders and injection-moulding machines. International Polymer Processing, 18, 226234 (2003).

[13] Miethlinger J.: Modelling the solids feed section in grooved-feed extruders. Kunststoffe Plast Europe, 93, 49-53 (2003).

[14] Rauwendaal C., Sikora J.: The adjustable grooved feed extruder. Plastics Additives and Compounding, 2, 2630 (2000).

DOI: $10.1016 / \mathrm{S} 1464-391 \mathrm{X}(00) 88882-0$

[15] Kowalska B.: Grooved feed sections: Design variants for single-screw extruders. Kunststoffe Plast Europe, 90, 10-11 (2000).

[16] Sikora J. W.: The effect of the feed section groove taper angle on the performance of a single-screw extruder. Polymer Engineering and Science, 41, 16361643 (2001). DOI: $10.1002 /$ pen.10861

[17] Rautenbach R., Peiffer H.: Model calculation for the design of the grooved feed section of single-screw extruders. Kunststoffe-German Plastics, 72, 137-143 (1982).

[18] Potente H.: The forced feed extruder must be reconsidered. Kunststoffe-German Plastics, 78, 355-363 (1988).

[19] Potente H., Schöppner V.: A throughput model for grooved bush extruders. International Polymer Processing, 10, 289-295 (1995).

[20] Rauwendaal C.: Polymer extrusion. Hanser, Munich (2010) 
[21] Potente H., Phol T. C.: Polymer pellet flow out of the hopper into the first section of a single screw. International Polymer Processing, 17, 11-21 (2001).

[22] Moysey P. A., Thompson M. R.: Investigation of solids transport in a single-screw extruder using a 3-D discrete particle simulation. Polymer Engineering and Science, 44, 2203-2215 (2004).

DOI: $10.1002 /$ pen.20248

[23] Moysey P. A., Thompson M. R.: Modelling the solids inflow and solids conveying of single-screw extruders using the discrete element method. Powder Technology, 153, 95-107 (2005).

DOI: $10.1016 /$ j.powtec.2005.03.001

[24] Moysey P. A., Thompson M. R.: Determining the collision properties of semi-crystalline and amorphous thermoplastics for DEM simulations of solids transport in an extruder. Chemical Engineering Science, 62, 3699-3709 (2007).

DOI: $10.1016 /$ j.ces.2007.03.033
[25] Michelangelli O. P., Yamanoi M., Gaspar-Cunha A., Covas J. A.: Modelling pellet flow in single extrusion with DEM. Journal of Process Mechanical Engineering, 225, 255-268 (2011).

DOI: $10.1177 / 0954408911418159$

[26] Chung C. I.: Plasticating single-screw extrusion theory. Polymer Engineering and Science, 11, 93-98 (1971).

DOI: $10.1002 /$ pen.760110204

[27] Qu J., Shi B., Feng Y., He H.: Dependence of solids conveying on screw axial vibration in single screw extruders. Journal of Applied Polymer Science, 102, 2998-3007 (2006).

DOI: $10.1002 / a p p .24658$

[28] Fang S., Chen L., Zhu F.: Studies on the theory of single screw plasticating extrusion. Part II: Non-plug flow solid conveying. Polymer Engineering and Science, 31, 1117-1122 (1991). DOI: $10.1002 /$ pen. 760311508 